\title{
FK778 Attenuates Cytomegalovirus- Enhanced Vein Graft Intimal Hyperplasia in a Rat Model
}

\author{
Geoffrey Kloppenburg ${ }^{\mathrm{a}}$ Rick de Graaf ${ }^{\mathrm{b}}$ Gert Grauls ${ }^{\mathrm{a}}$ Cathrien A. Bruggeman ${ }^{\mathrm{a}}$ \\ Johannes P. van Hooff ${ }^{c}$ Frank Stassen ${ }^{\text {a }}$ \\ Departments of a Medical Microbiology, ${ }^{\mathrm{b}}$ Radiology and ${ }^{\mathrm{C}}$ Internal Medicine, University Hospital Maastricht/ \\ Maastricht University, Maastricht, The Netherlands
}

\section{Key Words}

Vein graft • Intima hyperplasia $\cdot$ Cytomegalovirus · FK778

\begin{abstract}
Background: Venous grafts are commonly used to treat drug-resistant coronary artery disease, although long-term functionality is limited because of proliferation and migration of smooth muscle cells (SMC). As proliferating SMC are particularly susceptible for the stimulating effects of cytomegalovirus (CMV), we hypothesized that CMV infection may enhance cell proliferation and graft failure. Furthermore, we evaluated the potential of FK778 to prevent intimal hyperplasia. Apart from its antiviral properties, FK778 is a new immunosuppressive agent which may also affect SMC proliferation, making it an interesting drug to prevent (CMVenhanced) venous graft intimal hyperplasia. Methods: Epigastric vein-to-common femoral artery interposition grafts were placed in four groups of 10 rats each. Rats received either FK778 (oral treatment, $15 \mathrm{mg} / \mathrm{kg}$ ), were infected with CMV (1.25 $\times 10^{6}$ plaque-forming units) or were both treated and infected. Results: CMV infection resulted in a significant increase in intimal and medial cross-sectional area and medial wall thickness of the vein grafts. This effect was diminished by administration of FK778. Moreover, FK778 treatment alone resulted in a significant decrease in neointimal area and percentage of stenosis versus the control group. Conclusions: These data suggest a role of CMV in venous
\end{abstract}

graft failure. Also, our results suggest a prospective role for the new immunosuppressive drug FK778 in the prevention of (CMV-mediated) vein graft intimal hyperplasia.

Copyright $\odot 2009$ S. Karger AG, Basel

\section{Introduction}

Autologous vein grafts are commonly used for coronary artery bypass grafting, along with angioplasty, for the treatment of advanced arterial stenosis and drug-resistant angina pectoris. Although the initial results of venous grafts are successful, the symptoms tend to recur due to degeneration and occlusion of the graft. Early thrombosis and neointimal hyperplasia are thought to be the primary causes of graft failure. Intimal hyperplasia (IH), defined as the accumulation of smooth muscle cells (SMC) and extracellular matrix in the intimal component of the vein, is the major disease process in the venous graft within the first year. It has been strongly suggested that IH is associated with extensive endothelial denudation and destruction of venous grafts, resembling a response to injury as seen after PTCA often leading to restenosis of the denuded artery [1]. Previous studies indicated that infectious agents, and in particular cytomegalovirus (CMV), increase the risk of restenosis after percutaneous coronary intervention $[2,3]$ and coronary atherectomy [4]. In addition, we recently showed that 
CMV aggravates IH in rats by stimulating SMC proliferation in an arterial injury model [5].

Although multiple studies demonstrated a role for CMV in IH, a possible contribution of CMV in venous bypass failure received only limited attention so far and results have been inconclusive hitherto [6-9]. Therefore, we decided to elucidate the role of CMV on vein graft IH in an experimental animal model.

Moreover, we evaluated whether treatment with FK778 (i) could restrain vein graft stenosis or (ii) reduce the aggravating effects of CMV on IH due to its antiviral properties. The synthetic malononitrile amide FK778 (MNA 715 ) is one of the most interesting new immunosuppressive/antiproliferative drugs [10]. It is a derivative of A77 1726 , the active metabolite of leflunomide, which has already been shown to be effective in reducing rat aortic SMC proliferation [15]. FK778 binds specifically to the enzyme dihydroorotate dehydrogenase, thereby inhibiting the de novo pyrimidine biosynthesis [11] and consequently cell proliferation. Besides its effect on pyrimidine synthesis, FK778 may also affect other processes potentially involved in IH. For example, FK778 has been shown to reduce endothelial adhesion molecule upregulation and to attenuate lymphocyte-endothelium interaction [13]. In addition, FK778 effectively attenuated vascular response to injury by diminishing neointima formation through the inhibition of receptor tyrosine kinase activity in vascular SMC or their progenitors [14]. Furthermore, despite different modes of action, antiviral properties have been attributed to both FK778 and A77 1726 [16-18]. These vasculoprotective and antiviral properties of FK778 make it a potentially interesting drug to prevent not only venous graft $\mathrm{IH}$, but also to inhibit the aggravating effects of CMV infections on this process. Both issues were addressed in the present study.

\section{Materials and Methods}

\section{Animal Conditions and Vein Grafting Procedure}

Male inbred specific pathogen-free Lewis rats were obtained from the Department of Experimental Animal Service of the University of Maastricht, the Netherlands. Experiments were carried out on animals aged 12 weeks weighing 250-350 g. Housing and care of the animals, and all the procedures used in this study, were approved by the Ethical Committee for the Use of Experimental Animals of the institution, and conformed to the Guide for the Care and the Use of Laboratory Animals, published by the US National Institute of Health (NIH Publ. No. 85-23, revised 1985). Rats were fed standard rat chow and tap water at libitum. All surgical procedures were performed under general anesthesia using a sterile technique. Epigastric vein-to-common femoral artery in- terposition grafts were placed in rats by the use of a method similar to that described earlier by Hoch et al. [19]. In brief, each animal was anesthetized with an intraperitoneal injection of pentobarbital sodium $(60 \mathrm{mg} / \mathrm{kg})$. An $8-\mathrm{mm}$ segment of the ipsilateral epigastric vein was carefully harvested, gently irrigated with heparinized saline solution $(100 \mathrm{U} / \mathrm{ml})$, and placed as reverse interposition graft into a segmental 3-mm defect of the common femoral artery with 8-10 interrupted sutures of 11-0 nylon (Ethicon). The entire procedure was carried out with standard microsurgical techniques. The total ischemic time was kept to $<30 \mathrm{~min}$. Graft patency, defined as a condition of flow through the graft, was verified by visual inspection.

\section{Experimental Design}

The whole experiment consisted of a total of four experimental groups ( $\mathrm{n}=10$ animals/group) which all underwent surgery. Furthermore, rats were either infected with CMV, treated with FK778 or both. A control group only received the FK778 solvent (1 ml of a $1 \%$ carboxymethylcellulose solution in water). Infection was established by inoculating rats intraperitoneally with $1.25 \times 10^{6}$ plaque-forming units of homogenized salivary gland-derived rat CMV (RCMV, Maastricht strain) [20] immediately after surgery.

FK778 was supplied by Fujisawa Healthcare Inc. (Osaka, Japan). For oral administration, FK778 powder was suspended in $1 \%$ carboxymethylcellulose solution in water, until a final concentration of $5 \mathrm{mg} / \mathrm{ml}$ was reached. Animals received an oral dose of $15 \mathrm{mg} / \mathrm{kg}$ body weight per day from 1 day prior to the operation till the day of tissue harvest.

\section{Histological and Morphometrical Procedures}

Three weeks after surgery, rats were anesthetized and graft patency was verified by visual inspection. Then the chest and abdominal cavities were opened and a catheter was inserted into the apex of the heart. Vasculature was initially flushed with physiological salt solution and then perfusion-fixed with 3.7\% formaldehyde in phosphate-buffered saline (PBS), $\mathrm{pH}$ 7.4, at physiological pressure $(100 \mathrm{~mm} \mathrm{Hg})$. Vein grafts were removed and fixed overnight in the same fixative and routinely processed for paraffin embedding. Cross sections $(4 \mu \mathrm{m})$ were taken for morphometrical analysis and hematoxylin and eosin or Lawson stained (average of three cross sections per graft). Intimal and medial areas were quantified using a computer-assisted morphometry system (analySIS ${ }^{\circledR}$, Soft Imaging System, GmbH). The cross-sectional area of the media was defined as the area surrounded by the external and internal elastic lamina. The neointimal cross-sectional area was defined by the area surrounded by the internal elastic lamina and the arterial lumen. Next, the percentage of stenosis was calculated which is defined as the percentage of crosssectional area of the former lumen occluded by the neointima. Final scores were given as percentage of the control group.

\section{Quantification of the Number of Cells in the Intima and Media}

The number of cells in the intima and media was quantified by counting the total number of nuclei using a microscope. All nuclei were counted in hematoxylin and eosin stained cross sections taken from patent grafts in which a neointima was observed. Final scores were expressed as number of cells per $\mu \mathrm{m}^{2}$ and values are given as mean. 


\section{Immunohistochemistry}

Paraffin sections $(4 \mu \mathrm{m})$ taken from vein grafts 3 weeks after surgery were routinely processed and stained with the two-layer indirect immunoperoxidase technique using monoclonal antibodies (mAbs). The following mAbs were used in this study: antirat CD3 (Sera-Lab Ltd., Crawley Down, UK) and anti- $\alpha$-smooth muscle actin (Sigma, St. Louis, Mo., USA); ED-1, a mouse mAb to monocytes/macrophages [21], was kindly supplied by Dr. A.M. Duijvestijn (Department of Immunology, University Maastricht, the Netherlands).

Sections were incubated with 2\% BSA in PBS (ED-1 and $\alpha$-actin) for $15 \mathrm{~min}$ at room temperature and treated with antigen retrieval buffer (CD3) for $25 \mathrm{~min}$ at $95^{\circ}$ [22]. $\mathrm{mAb}$ were diluted (ED$11 / 20, \alpha$-actin $1 / 1,500$ and CD3 1/400) in PBS and applied to the slides for $60 \mathrm{~min}$ at $37^{\circ}$. After 3 washes with PBS for $5 \mathrm{~min}$, a biotinylated goat anti-mouse secondary antibody (1/1,200, Dako, Glostrup, Denmark) was applied for $30 \mathrm{~min}$ at room temperature. Finally, sections were incubated with alkaline phosphatase-coupled streptavidin (ABC reagent, Vector Laboratories), followed by immunodetection using Fast Red as a substrate. Then the presence of positive cells was analyzed by microscopy and scored semiquantitatively ( 0 = no staining, $1=$ positive staining, $2=$ extensive positive staining) by an observer blinded to the specimen group.

\section{Statistical Analysis}

All data were expressed as mean \pm SEM. To analyze differences for statistical significance, a Mann-Whitney U test was performed using SPSS 11.0 for Windows (SPSS, Chicago, Ill., USA). Differences were considered to be statistically significant when $\mathrm{p}$ values were $<0.05$.

\section{Results}

\section{Animal Condition and Grafts}

No apparent clinical signs of illness were observed in any of the animals during the follow-up period. Starting body weight ranged from 250 to $350 \mathrm{~g}$; at the end of the experiments animal weight ranged from 325 to $405 \mathrm{~g}$. The overall graft patency was $90 \%$. There were no significant differences in body weights or graft patency between the experimental groups (data not shown). There were no structural abnormalities at the anastomotic regions of the grafts. The gross appearance of the veins and adjoining arteries was normal.

\section{FK778 Attenuates CMV-Aggravated Intimal Hyperplasia}

Three weeks after bypass grafting the medial crosssectional area was increased and a pronounced neointimal thickening was observed in all grafted veins (fig. 1, 2a). CMV infection immediately after vein grafting resulted in a significant increase in intimal cross-sectional area, medial cross-sectional area and medial wall thickness at 3 weeks after surgery as compared to the control
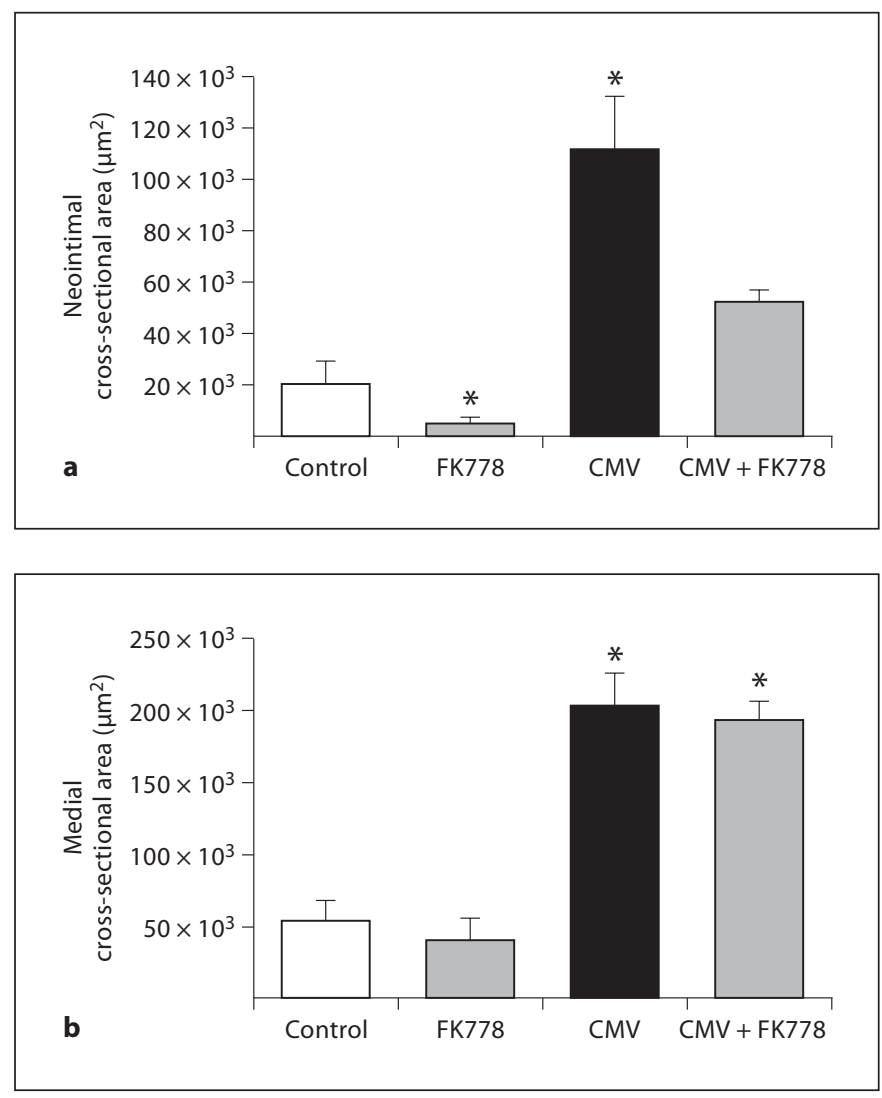

Fig. 1. Effect of FK778, CMV or co-administration of CMV and FK778 on neointimal (a) and medial (b) cross-sectional areas 3 weeks after vein grafting. ${ }^{*} \mathrm{p}<0.05$ vs. controls.

group (fig. 1, 2b). This effect was partially reversed by FK778 treatment, resulting in a significant decrease of the CMV-aggravated neointimal cross-sectional area as well as percentage of stenosis but not medial cross-sectional area or medial thickness. Furthermore, FK778 treatment alone resulted in a significant decrease in neointimal area and percentage of stenosis as compared to the control group, although no effects were seen on medial cross-sectional area or medial thickness (fig. 1, table 2).

\section{Quantification of Number of Cells in the Intima and Media}

Although the medial and intimal cross-sectional area was significantly increased at 3 weeks when rats were infected immediately after surgery, the average number of cells $/ \mu \mathrm{m}^{2}$ was not different as compared to the control group (table 1). This implies that the total number of cells in the intima and media is increased and suggests that CMV predominantly stimulated cell proliferation. FK778 


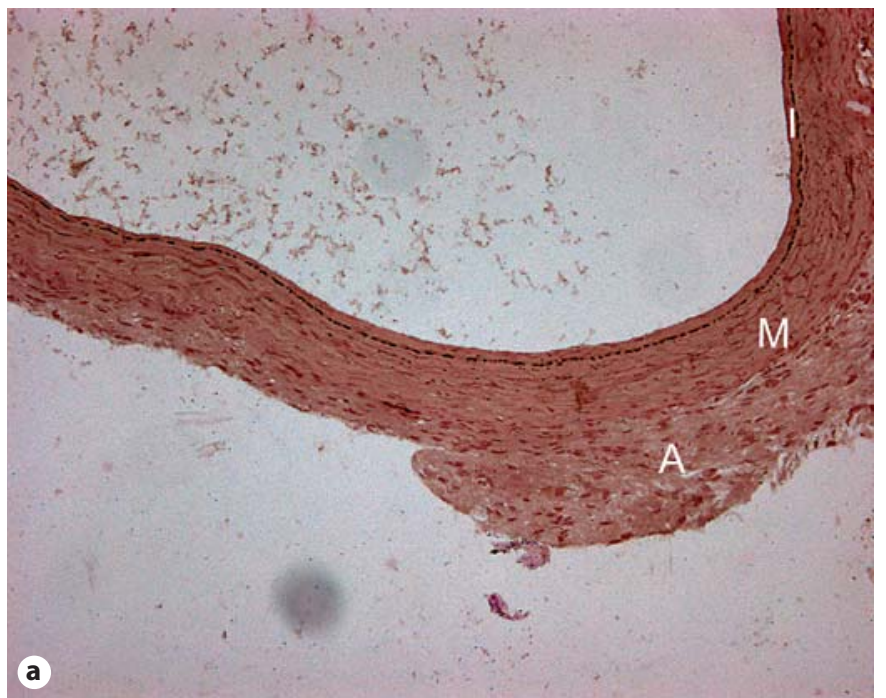

Fig. 2. Typical examples of venous graft cross sections 21 days after grafting. a Lawson staining venous graft control group. b Lawson staining venous graft CMV-infected group. c ED-1 monocyte macrophage staining. $\mathrm{I}=$ Intima; $\mathrm{M}=$ media; $\mathrm{A}=$ adventitia.

Table 1. Cellular density in both media and neointima of all groups

\begin{tabular}{lrrrr}
\hline & Control & \multicolumn{1}{l}{ CMV } & \multicolumn{1}{l}{ FK778 } & \multicolumn{1}{c}{ CMV+FK778 } \\
\hline Media & $4.4 \pm 0.7$ & $4.9 \pm 0.6$ & $4.3 \pm 0.6$ & $4.7 \pm 0.3$ \\
Neointima & $16.4 \pm 3.5$ & $15.4 \pm 3.6$ & $18.1 \pm 2.1$ & $16.5 \pm 3.1$ \\
\hline
\end{tabular}

Data are expressed as $\times 10^{-3}$ cells $/ \mu \mathrm{m}^{2}$. No significant differences were observed between groups.

treatment resulted in a decrease of intimal cross-sectional area, but again the average number of cells $/ \mu \mathrm{m}^{2}$ was not different as compared to the control group (table 1). This implies that the total number of cells in the intima
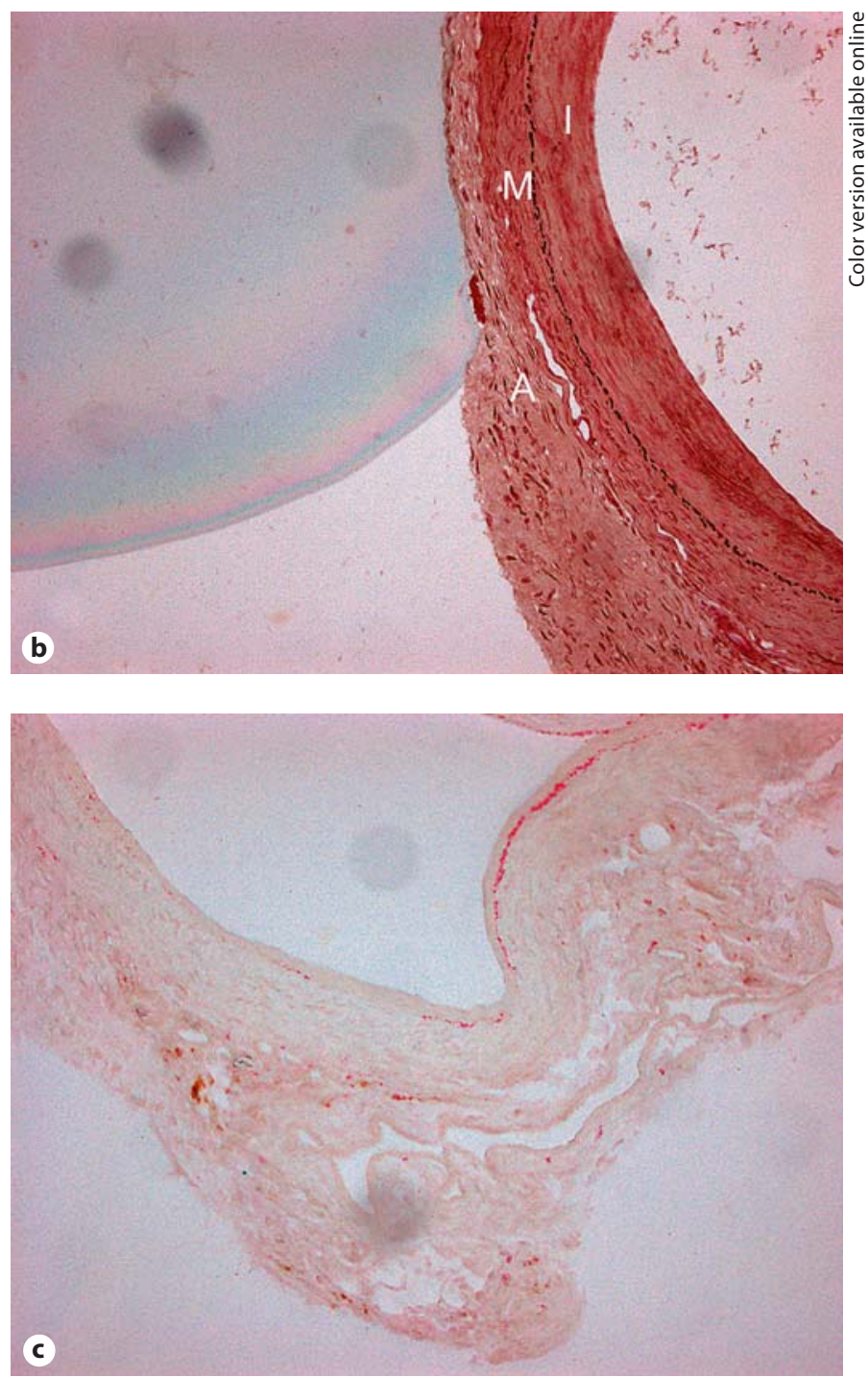

is decreased and suggests that cell proliferation has been attenuated by FK778.

\section{Immunohistochemistry}

The number of T cells in the venous graft 3 weeks after surgery was minimal with an average of 1-3 T cells per cross section. No difference in T-cell number/section was observed between groups. Presence of macrophages was abundant and cells were most frequently detected in the subendothelial space as well as in the intima (fig. 2c). However, when stainings were analyzed in a semiquantitative way, no significant differences were noted between groups (data not shown). $\alpha$-Actin staining showed SMC to be the main content of the intima and media with little extracellular matrix accumulation in all groups. 
Table 2. Summary of morphological alterations found in CMV-infected rats

\begin{tabular}{|c|c|c|c|c|}
\hline & Intimal area, $\mu \mathrm{m}^{2}$ & Medial area, $\mu \mathrm{m}^{2}$ & $\begin{array}{l}\text { Stenosis } \\
\%\end{array}$ & $\begin{array}{l}\text { Wall thickness } \\
\mu \mathrm{m}\end{array}$ \\
\hline CMV & ${ }^{*} 112 \times 10^{3} \pm 19 \times 10^{3}$ & ${ }^{*} 203 \times 10^{3} \pm 21 \times 10^{3}$ & $32 \pm 13$ & $* 77 \pm 9.0$ \\
\hline $\mathrm{CMV}+\mathrm{FK} 778$ & $53 \times 10^{3} \pm 4.5 \times 10^{3}$ & ${ }^{*} 193 \times 10^{3} \pm 13 \times 10^{3}$ & $12 \pm 1.2$ & $* 59 \pm 4.0$ \\
\hline FK778 & ${ }^{*} 5.8 \times 10^{3} \pm 2.6 \times 10^{3}$ & $41 \times 10^{3} \pm 14 \times 10^{3}$ & $* 8.8 \pm 2.5$ & $42 \pm 12$ \\
\hline Control & $21 \times 10^{3} \pm 8.2 \times 10^{3}$ & $54 \times 10^{3} \pm 13 \times 10^{3}$ & $* 52 \pm 17$ & $36 \pm 3.3$ \\
\hline
\end{tabular}

FK778-treated rats or CMV-infected/FK778-treated animals 3 weeks after vein grafting.

${ }^{*} \mathrm{p}<0.5$ compared to data obtained in control rats.

\section{Discussion}

Venous graft failure is a common problem, occurring in $10-15 \%$ of grafts during the first year, increasing up to $40 \%$ within 10 years [23]. Early thrombosis and neointimal hyperplasia are considered to be the primary causes of graft failure. Nonetheless, although a research subject for several decades, the exact underlying mechanism of venous graft failure has not been elucidated yet. As CMV infections have been shown to accelerate arterial restenosis, we hypothesized that the virus may also contribute to venous graft failure. This was addressed in the present study in which we investigated whether CMV contributes to venous graft failure in a rat epigastric vein-to-common femoral artery graft model. In addition, we studied the therapeutic potential of the immunosuppressive/antiproliferative drug FK778 in preventing IH with or without a concomitant CMV infection. Here we present for the first time evidence that CMV contributes to the process of venous graft failure. Furthermore, we demonstrate that FK778 is able to partially prevent IH in a venous bypass graft in non-infected animals, while the drug was also able to reverse the additional effect of a concomitant CMV infection on IH (table 2).

Neointimal hyperplasia, defined as the accumulation of SMC and extracellular matrix in the intimal compartment of blood vessels, is the major disease process in venous grafts within the first year. Nearly all veins implanted into an arterial circulation develop intimal wall thickening, thereby reducing the lumen size. This is confirmed by our results in the rat venous graft model. In the epigastric vein-to-common femoral artery interposition grafts a significant enlargement of the vascular wall was observed at 3 weeks posttransplantation, which is in agreement with earlier data presented by others [19].

This intimal wall thickening predominantly results from excessive SMC proliferation in response to injury.
Under normal conditions, SMC in the media of blood vessels proliferate at very low rates, but can switch very rapidly from quiescence to a proliferative state in response to appropriate stimuli like bypass grafting or angioplasty [24]. Previously it has been shown that CMV accelerates cellular proliferation [25]. This suggestion is supported by the present data. Although the total intimal area was significantly increased following CMV infection, the average number of cells per $\mu \mathrm{m}^{2}$ was unchanged, indicating that CMV stimulates SMC proliferation but not cellular hypertrophy or extensive accumulation of extracellular matrix ultimately resulting in an enlarged medial and intimal area. The latter was confirmed by $\alpha$ actin immunostaining showing that the majority of the intima and media consisted of SMC. These results are in accordance with earlier data from our group, in which we demonstrated that CMV stimulates SMC proliferation in the thrombosis-induced intimal thickening in the rat femoral artery [5]. Although the exact mechanism is unclear until now, it has previously been suggested that inhibition of the p53 suppressor effects are the predominant cause of CMV-induced stimulation of SMC proliferation [25]. Nevertheless, additional research is required to further clarify the effect of CMV on cellular proliferation.

The new malononitrile amide FK778 is currently being evaluated as an immunosuppressant for organ transplantations as it effectively inhibits T-cell proliferation and alloantibody synthesis by B cells [12]. Furthermore, besides being immunosuppressive, vasculoprotective properties have also been attributed to FK778. More precisely, it has been shown that FK778 may inhibit SMC proliferation. In various experimental models it has been demonstrated that FK778 effectively attenuates vascular responses to injury by diminishing neointima formation and fibrointimal hyperplasia, perhaps through inhibition of the receptor tyrosine kinase activity in vascular 
SMC or their progenitors $[14,27,28]$. Also in our rat model of epigastric vein-to-common femoral artery interposition grafts a daily regimen of $15 \mathrm{mg} / \mathrm{kg}$ FK778 significantly reduced $\mathrm{IH}$ resulting in a lower percentage stenosis as compared to the control group. Quantification of the number of cells per $\mu \mathrm{m}^{2}$ revealed no significant differences between groups, indicating that the reduction in cross-sectional intimal area caused by FK778 is due to a decrease in SMC proliferation.

Along with its antiproliferative activity, antiviral properties against CMV have been assigned to FK778 [17, 18]. FK778 is a potent and effective inhibitor of CMV and its mode of antiviral action appears to mirror the biochemical mechanism described to be responsible for its immunosuppressive properties: inhibition of protein tyrosine phosphorylation and inhibition of cellular de novo pyrimidine biosynthesis [16]. Here we demonstrate that FK778 is able to partially prevent the CMV-mediated enhancement of $\mathrm{IH}$ in the venous graft. Treatment with FK778 significantly reduced the effect of CMV on intimal area as well as on the percentage stenosis. In contrast, no effects of FK778 treatment were observed on the medial cross-sectional area.

It remains to be established whether the antiviral or the antiproliferative properties of FK778 are predominantly responsible for its inhibitory effect on the CMVmediated enhancement of intima formation in infected animals. Previously, we demonstrated that CMV aggravates $\mathrm{IH}$ in a thrombosis-induced intimal thickening model [5] mainly by stimulating SMC proliferation. As hardly any virus could be detected in the vascular wall at various stages of the hyperplastic process, it was suggested that inflammatory and immune responses are more important in the CMV-mediated augmentation of the hyperplastic response than the virus itself. Based on these findings we assume that the effects of FK778 in our venous graft model should not be ascribed to its antiviral characteristics.

Although inhibition of the cellular and humoral immune response is of great importance in preventing acute rejection and also chronic allograft dysfunction in transplantation, it is questionable whether it plays a significant role in venous graft failure. Hoch et al. [19] showed the relative absence of CD4+ and CD8+ cells in the epigastric vein-to-common femoral artery interposition grafts in the rat model. Likewise, we also found only a limited number of $\mathrm{CD} 3+$ cells in the grafts at 3 weeks after surgery with no clear difference between the infected, treated and the control group. This also suggests that it is unlikely that the effect of FK778 on intima hyperplasia is due to its immunosuppressive activity. Alternatively, it has recently been shown that FK778 directly reduces ICAM-1 and VCAM-1 upregulation and attenuates lymphocyte-endothelium interaction [13]. We therefore speculated that administration of FK778 might result in a decreased influx of macrophages in our venous grafts. However, immunological staining showed no clear differences in the influx of macrophages in the grafts at 3 weeks after surgery. In conclusion, the majority of our data suggest that the effect of FK778 on intima hyperplasia has mainly to be attributed to its effect on SMC proliferation.

In conclusion, in a well-defined animal model, we present for the first time evidence for a possible role of $\mathrm{CMV}$ in the process of venous graft failure, which could partially be inhibited by the new immunosuppressive agent FK778. These data suggest that FK778 might be a potential candidate to prevent $\mathrm{IH}$ following venous bypass surgery.

\section{References}

1 LoGerfo FW, Quist WC, Cantelmo NL, et al: Integrity of vein grafts as a function of initial intimal and medial preservation. Circulation 1983;68:II117-II124.

-2 Horne BD, Muhlestein JB, Strobel GG, et al; Intermountain Heart Collaborative (IHC) Study Group: Greater pathogen burden but not elevated C-reactive protein increases the risk of clinical restenosis after percutaneous coronary intervention. Am Heart J 2002;144: 491-500.
-3 Mueller C, Hodgson JM, Bestehorn HP, et al: Cytomegalovirus infection and restenosis after aggressive angioplasty with provisional stenting. J Interv Cardiol 2003;16:307-313.

4 Zhou YF, Leon MB, Waclawiw MA, et al: Association between prior cytomegalovirus infection and the risk of restenosis after coronary atherectomy. N Engl J Med 1996;29; 335:624-630.

5 Kloppenburg G, de Graaf R, Herngreen S, et al: Cytomegalovirus aggravates intimal hyperplasia in rats by stimulating smooth muscle cell proliferation. Microbes Infect 2005;7: 164-170.

\footnotetext{
6 Bartels C, Maass M, Bein G, et al: Detection of Chlamydia pneumoniae but not cytomegalovirus in occluded saphenous vein coronary artery bypass grafts. Circulation 1999; 99:879-882.

7 Bartels C, Maass M, Bein G, et al: Association of serology with the endovascular presence of Chlamydia pneumoniae and cytomegalovirus in coronary artery and vein graft disease. Circulation 2000;101:137-141.
} 
$>$ Ibrahim AI, Obeid MT, Jouma MJ, et al: Detection of herpes simplex virus, cytomegalovirus and Epstein-Barr virus DNA in atherosclerotic plaques and in unaffected bypass grafts. J Clin Virol 2005;32:29-32.

-9 Limnell V, Pasternack R, Karjalainen J, et al: Seropositivity for Helicobacter pylori antibodies is associated with lower occurrence of venous bypass graft occlusion. Scand J Infect Dis 2004;36:601-603.

10 Vincenti F: What's in the pipeline? New immunosuppressive drugs in transplantation. Am J Transplant 2002;2:898-903.

-11 Silva HT Jr, Cao W, Shorthouse RA, et al: In vitro and in vivo effects of leflunomide, brequinar, and cyclosporine on pyrimidine biosynthesis. Transplant Proc 1997;29:12921293.

12 Kurrle R, Bartlett R, Ruuth E, et al: Malononitrilamides inhibit $\mathrm{T}$ - and $\mathrm{B}$-cell responsiveness. Transplant Proc 1996;28:30533056.

13 Deuse T, Schrepfer S, Schafer H, et al: FK778 attenuates lymphocyte-endothelium interaction after cardiac transplantation: in vivo and in vitro studies. Transplantation 2004; 78:71-77.

-14 Savikko J, Von Willebrand E, Hayry P: Leflunomide analogue FK778 is vasculoprotective independent of its immunosuppressive effect: potential applications for restenosis and chronic rejection. Transplantation 2003; 76:455-458.
15 Czech J, Kurrle R, Schorlemmer HU: The antiproliferative effect of malononitrilamides on vascular smooth muscle cells is antagonized by exogenous uridine. Inflamm Res 1999;48(suppl 2):S128-S129.

16 Evers DL, Wang X, Huong SM, et al: Inhibition of human cytomegalovirus signaling and replication by the immunosuppressant FK778. Antiviral Res 2005;65:1-12.

17 Waldman WJ, Knight DA, Lurain NS, et al: Novel mechanism of inhibition of cytomegalovirus by the experimental immunosuppressive agent leflunomide. Transplantation 1999;68:814-825.

18 Waldman WJ, Knight DA, Blinder L, et al: Inhibition of cytomegalovirus in vitro and in vivo by the experimental immunosuppressive agent leflunomide. Intervirology 1999; 42:412-418.

19 Hoch JR, Stark VK, Hullett DA, et al: Vein graft intimal hyperplasia: leukocytes and cytokine gene expression. Surgery 1994;116 463-470.

20 Bruggeman CA, Meijer H, Dormans PH, et al: Isolation of a cytomegalovirus-like agent from wild rats. Arch Virol 1982;73:231-241.

21 Dijkstra CD, Dopp EA, Joling P, Kraal G: The heterogeneity of mononuclear phagocytes in lymphoid organs: distinct macrophage subpopulations in the rat recognized by monoclonal antibodies ED1, ED2 and ED3. Immunology 1985;54:589-599.

22 McKechnie NM, Gurr W, Braun G: Immunization with the cross-reactive antigens Ov39 from Onchocerca volvulus and hr44 from human retinal tissue induces ocular pathology and activates retinal microglia. J Infect Dis 1997;176:1334-1343.
23 Fitzgibbon GM, Kafka HP, Leach AJ, et al: Coronary bypass graft fate and patient outcome: angiographic follow-up of 5,065 grafts related to survival and reoperation in 1,388 patients during 25 years. J Am Coll Cardiol 1996;28:616-626.

24 Majesky MW, Schwartz SM, Clowes MM, et al: Heparin regulates smooth muscle $S$ phase entry in the injured rat carotid artery. Circ Res 1987;61:296-300.

25 Speir E, Modali R, Huang ES, et al: Potential role of human cytomegalovirus and p53 interaction in coronary restenosis. Science 1994;265:391-394.

26 Persoons MC, Daemen MJ, Bruning JH, et al: Active cytomegalovirus infection of arterial smooth muscle cells in immunocompromised rats. A clue to herpesvirus-associated atherogenesis? Circ Res 1994;75:214-220.

27 Jahnke T, Schafer FK, Bolte H, et al: 2005 Dr. Gary J. Becker Young Investigator Award: periprocedural oral administration of the leflunomide analogue FK778 inhibits neointima formation in a double-injury rat model of restenosis. J Vasc Interv Radiol 2005; 16:903-910.

28 Pan F, Ebbs A, Wynn C, et al: FK778, a powerful new immunosuppressant, effectively reduces functional and histological changes of chronic rejection in rat renal allografts. Transplantation 2003;75:1110-1114. 